\title{
Systematic Review and Meta-analysis on COVID-19 Vaccine Hesitancy
}

Iman Ahmed Fathalla Aboelsaad ${ }^{1}$,Dina Mohamed $\mathrm{Hafez}^{2}$,Abdallah Almaghraby ${ }^{3}$ Shaimaa Abdulaziz Abdulmoneim ${ }^{1}$, Samar Ossama El-ganainy ${ }^{4}$, Noha Alaa Hamdy ${ }^{5}$, Ehsan Akram Deghidy $^{6}$, Ahmed El-Sayed Nour El-Deen ${ }^{7}$, Ehab Mohamed Adel Elrewany ${ }^{8}$, Alaa Hamdy Taha Khalil $^{9}$, Karem Mohamed Salem ${ }^{10}$, Samar Galal kabeel ${ }^{1}$, Yasir Ahmed Mohammed Elhadi ${ }^{11}$, Ramy Shaaban ${ }^{12}$, Amr Alnagar ${ }^{13}$,Eman Ahmad Fadel Elsherbeny ${ }^{14}$, Nagwa Ibrahim Elfeshawy ${ }^{14}$, Mohamed Moustafa Tahoun ${ }^{15}$,Ramy Mohamed Ghazy ${ }^{16}$

1 Egyptian Ministry of Health and Population

2 Pharmacy Section, Alexandria University Students Hospital, Epidemiology Specialist, Alexandria University, Egypt

3 Department of Cardiology and Angiology, Alexandria University, Egypt

4 Department of Pharmacology \& Therapeutics, Faculty of Pharmacy, Pharos University in Alexandria

5 Pharmacy Practice Department, Faculty of Pharmacy, Alexandria University

6 Department of Biomedical Informatics and Medical Statistics, Medical Research Institute, Alexandria University, Alexandria,

7 Department of Physiology, Faculty of Medicine, Al-Azhar University, Assuit, Egypt.

8 Tropical Health Department, Hiph Institute of Public Health, Alexandria University

9 Physician, Cairo University Medical School

10 Department of internal medicine, Faculty of medicine, Fayoum University, Egypt

11 Department of Public Health, Medical Research office, Sudanese Medical Research Association, Khartoum, Sudan

12 Department of Instructional Technology and Learning Sciences, Utah State University, USA

13Department of General Surgery-Faculty of Medicine-Alexandria University

NOTE: This preprint reports new research that has not been certified by peer review and should not be used to guide clinical practice. 
medRxiv preprint doi: https://doi.org/10.1101/2021.05.15.21257261; this version posted May 18, 2021. The copyright holder for this preprint (which was not certified by peer review) is the author/funder, who has granted medRxiv a license to display the preprint in perpetuity.

It is made available under a CC-BY 4.0 International license .

14 Department of Woman's Health and Midwifery Nursing, Faculty of Nursing, Mansoura University, Egypt

15 World Health Organization, Regional Office for The Eastern Mediterranean - WHO Health Emergencies Programme, Department of Epidemiology, High Institute of Public Health, Alexandria University, Egypt

16 Tropical Health Department, High Institute of Public Health, Alexandria University Egypt

*Corresponding author: E-mail: dr.maghraby@gmail.com

Tel: 01222851687

Running Title:Systematic Review and Meta-analysis on COVID-19 Vaccine Hesitancy 


\begin{abstract}
Background:

The presented meta-analysis was developed in response to the publication of several studies addressing COVID-19 vaccines hesitancy. We aimed to identify the proportion of vaccine acceptance and rejection, and factors affecting vaccine hesitancy worldwide especially with the fast emergency approval of vaccines.
\end{abstract}

\title{
Methods:
}

Online database search was performed, and relevant studies were included with no language restriction. A meta-analysis was conducted using $\mathrm{R}$ software to obtain the random effect model of the pooled prevalence of vaccine acceptance and rejection. Egger's regression test was performed to assess publication bias. Quality assessment was assessed using Newcastle-Ottawa Scale quality assessment tool.

\section{Results:}

Thirty-nine out of 12246 articles met the predefined inclusion criteria. All studies were cross-sectional designs. The pooled proportion of COVID-19 vaccine hesitancy was 17\% (95\% CI: 14-20) while the pooled proportion of COVID-19 vaccine acceptance was 75\% (95\% CI: 71-79). The vaccine hesitancy and the vaccine acceptance showed high heterogeneity $\left(\mathrm{I}^{2}=100 \%\right)$. Case fatality ratio and the number of reported cases had significant effect on the vaccine acceptance as the pooled proportion of vaccine acceptance increased by 39.95\% (95\% CI: 20.1-59.8) for each $1 \%$ increase in case fatality $(\mathrm{P}<0.0001)$ and decreased by $0.1 \%(95 \% \mathrm{CI}:-0.2-0.01)$ for each 1000 reported case of COVID-19, $\mathrm{P}=0.0183$ ).

\section{Conclusion:}

Transparency in reporting the number of newly diagnosed COVID-19 cases and deaths is mandatory as these factors are the main determinants of COVID-19 vaccine acceptance.

Keywords: COVID-19, Vaccine hesitancy, vaccine side effects, vaccine immunogenicity, vaccine intake, vaccine acceptance

\section{Abbreviations:}

\begin{tabular}{|l|l|}
\hline AESI & $\begin{array}{l}\text { Adverse Events of Special } \\
\text { Interest }\end{array}$ \\
\hline
\end{tabular}




\begin{tabular}{|l|l|}
\hline CAWI & $\begin{array}{l}\text { Computer Assisted Web } \\
\text { Interviews }\end{array}$ \\
\hline CI & Confidence Interval \\
\hline COVID-19 & Coronavirus Disease \\
\hline F & Female \\
\hline FDA & $\begin{array}{l}\text { Food and Drug } \\
\text { Administration }\end{array}$ \\
\hline HCWs & Health Care Workers \\
\hline M & Male \\
\hline mRNA & $\begin{array}{l}\text { Messenger Ribonucleic } \\
\text { Acid }\end{array}$ \\
\hline n & Number \\
\hline NA & Not Available \\
\hline PRISMA & $\begin{array}{l}\text { Preferred Reporting Items } \\
\text { of Systematic Review and } \\
\text { Meta-analysis }\end{array}$ \\
\hline QA & Quality Assessment \\
\hline RRR & Relative Risk Ratio \\
\hline SARS-CoV-2 & $\begin{array}{l}\text { Severe Acute Respiratory } \\
\text { Syndrome Coronavirus 2 }\end{array}$ \\
\hline SD & Standard Deviation \\
\hline SMS & Short Message Service \\
\hline UK & United Kingdom \\
\hline US & United States \\
\hline VAERS & $\begin{array}{l}\text { Vaccine Adverse Event } \\
\text { Reporting System }\end{array}$ \\
\hline VPD & $\begin{array}{l}\text { Vaccine Preventable } \\
\text { Diseases }\end{array}$ \\
\hline WHO & World Health Organization \\
\hline & \\
\hline
\end{tabular}


medRxiv preprint doi: https://doi.org/10.1101/2021.05.15.21257261; this version posted May 18, 2021. The copyright holder for this preprint

\section{Introduction:}

The wide use of vaccines has led to decreased mortality and morbidity of different transmissible diseases, this was a crucial factor in elimination of poliomyelitis in the Americas and the worldwide eradication of smallpox (1). Vaccination programs depend on mass vaccination to be able to decrease incidence and prevalence of Vaccine Preventable Diseases (VPD). In addition to the proposed direct protection for vaccinated candidates, wide vaccination scope results in indirect shielding for the overall community by declined conveyance of VPD, thereby dampening the risk of infection for vulnerable individuals in the community (2).

One of the main limiting factors for wide-spread of vaccination programs (especially for newly emerging vaccines) is vaccine hesitancy. The World Health Organization (WHO) named vaccine hesitancy as one of the top ten threats to global health in 2019, calling for research to identify the factors associated with this phenomenon (3). Vaccine hesitancy is defined as a behavior of a delayed vaccine approval or even declined vaccination despite accessible vaccination services $(4,5)$.

The pandemic COVID-19 caused by the recently discovered coronavirus-2019 (SARS-CoV-2) is strongly influencing the worldwide public health, culture, economy, and human social behavior. Despite all efforts since the beginning of the pandemic there is no approved medicine or treatment to cure COVID-19 till now, whereas vaccine development efforts are taking the highest priority as it can potentially save humanity by inducing immunity against COVID-19 (6).

According to WHO, herd immunity against COVID-19, which is known as population immunity, can be achieved naturally by the exposed people who recovered from the virus by their own protective antibodies or by providing COVID-19 vaccination $(7,8)$. Herd immunity for COVID-19 can be achieved on $70 \%$ of the single vaccinated dose individuals and $90 \%$ of the two vaccinated dose individuals (9).

Vaccines typically require years of research and testing before reaching the clinic, but in 2020, scientists were racing against time to produce safe and effective coronavirus vaccines. Currently we have 14 approved vaccines for full use, 6 authorized in early or 
medRxiv preprint doi: https://doi.org/10.1101/2021.05.15.21257261; this version posted May 18, 2021. The copyright holder for this preprint

limited use, 27 vaccines in phase 3 trials, 36 vaccines in phase 2, 48 vaccines in phase 1 and 4 abandoned vaccines after trials. In addition, at least 77 preclinical vaccines are under active investigation in animals (10). Unfortunately, the newly emerging vaccines for COVID-19 are faced nowadays with hesitancy to use in different countries. People showed concerns about both efficacy and possible side effects of these recently approved vaccines. Such hesitancy can have a heavy influence on vaccine delivery and the aimed wide uptake to control the pandemic (11). After the announcement of several pharmaceutical manufactures the production of COVID-19 vaccines, social media started to discuss vaccine content widely across different platforms. The propagated information provides mostly non-factual data and from non-medical individuals (12).

The presented systematic review \& meta-analysis was developed in response to the publication of several studies addressing COVID-19 vaccines hesitancy. Identification of independent factors affecting vaccine hesitancy worldwide especially with the fast emergency approval of these vaccines.

\section{Methods}

\section{Data sources}

This meta-analysis was guided by the 2020 Cochrane Handbook of Systematic Review and Meta-Analysis (13), with respect to the preferred reporting items of the systematic review and meta-analysis (PRISMA) checklist (14). Search was conducted for the hesitancy or refusal of COVID-19 vaccination through the published and grey literature using multiple databases; PsycINFO, ScienceDirect, Embase, Scopus, EBSCO, MEDLINE central/PubMed, ProQuest, SciELO, SAGE, Web of science, and Google scholar. Search terms were determined and approved after the consultation of PubMed help desk. The used keywords were added to Annex 1.

\section{Study selection}

All studies reporting COVID-19 vaccine hesitancy, were included with no language restriction. Abstract-only papers as proposals, conference, editorials, author 
medRxiv preprint doi: https://doi.org/10.1101/2021.05.15.21257261; this version posted May 18, 2021. The copyright holder for this preprint

responses, reviews, case reports, case series, books and studies with data not accurately or reliably extracted, duplicate, or overlapping data were excluded.

\section{Data extraction and selection process}

Figure(1) depicts the PRISMA flow chart for the different steps of the systematic review. All articles were imported into EndNote X7.0.1 to detect and remove the duplicates with two methods: title, author, year, and then manually using title, author, and journal. Title and Abstract screening followed by full text screening were done after the citation's exportation to an Excel sheet containing the author's name, publication year, journal, DOI, URL link, and the abstract. Screening was performed independently by 3 reviewers NA Hamdy, EAD fourth reviewer IAA solved any disagreement. The kappa test of agreement between reviewers was 0.89 .

Further manual search for eligible citations was done through careful examination of the references of included studies as well as studies citing the selected articles using PubMed and google scholar. All included articles were extracted to an excel sheet with the following predefined data: year of publication, authors name, country, study design, study setting, study population, sample size, duration of the study, inclusion and exclusion criteria, percent refusal, percent acceptance, cause of refusal and cause of acceptance. Excel sheets are available online for reviewers. At Annex 2 https://drive.google.com/file/d/12tiK2yW9RGmVnxOTphDKOAuAFyMVub K/view?us $\mathrm{p}=$ sharing

\section{Investigations of heterogeneity:}

Cochrane $\mathrm{Q}$ test and $\left(\mathrm{I}^{2}\right)$ test was used to assess and measure heterogeneity between studies, considering $\mathrm{I}^{2} \geq 75 \%$ represents substantial heterogeneity and strength of evidence for heterogeneity is the $\mathrm{P}$ - value $\leq 0.05$ from the $\mathrm{Q}$ test; according to Cochrane Handbook for Systematic Reviews of Interventions (13). Due to substantial heterogeneity, DerSimonian and Laird random-effects models were applied to pool the outcomes.

\section{Publication bias:}


medRxiv preprint doi: https://doi.org/10.1101/2021.05.15.21257261; this version posted May 18, 2021. The copyright holder for this preprint

Publication biases were assessed by visual inspection of the funnel plot and statistically by Begg's modified funnel plot and Egger's regression test (13).

\section{Quality assessment}

Quality assessment (QA) was assessed using Newcastle-Ottawa Scale quality assessment tool customized for cross-sectional studies (15). The assessment was performed by two independent reviewers (DMH, EE) and further checked by two additional reviewers (SO EI-ganainy, AA).

\section{Statistical analysis and data synthesis:}

$\mathrm{R}$ software was used to perform the meta-analysis and to pool the effect size (proportion); fixed or random effect model were used according to the studies' consistency. Meta-regression analysis was performed to examine the impact of confounders on the effect of vaccine hesitancy such as age, sex, and country. Results were presented in the Forest plots to visualize the degree of variation between studies.

Leave one-out sensitivity analysis was conducted to test the effect of each study on the pooled effect to determine the robustness of the obtained outcomes. Sub-group analysis was performed to categorize the vaccine hesitancy according to sample size studies.

To investigate the sources of high heterogeneity in the pooled prevalence of vaccine acceptance and hesitancy, meta-regression analysis was performed with different models including the main predictors of vaccine acceptance and hesitancy reported in included studies such as age, sex, educational level and setting. Additionally, number of reported cases, number of reported deaths, case fatality ratio and number of vaccinated people within each country until the end of January $2021(16,17)$, were examined as potential modifiers of vaccine acceptance and hesitancy and included in the meta-regression model. 


\section{Results:}

\section{Search results:}

The flow diagram of the selection process is shown in figure 1. From a total of 12246 potentially relevant articles, 1621 duplicate articles and 2944 citations published before 2019 were excluded. A total of 7681 citations were eligible for title screening. Only 51 articles were eligible for full-text screening after removing irrelevant (7627) and duplicate articles (3). In total 34 articles were excluded after full text screening (2 duplicates and 29 irrelevant), 3 were retracted. Another 22 articles were added manually For quantitative assessment, there were 39 eligible articles. The inter-rater agreement for inclusion was $\kappa=0.87$ and for the quality assessment was $\kappa=0.91$. 
medRxiv preprint doi: https://doi.org/10.1101/2021.05.15.21257261; this version posted May 18, 2021. The copyright holder for this preprint (which was not certified by peer review) is the author/funder, who has granted medRxiv a license to display the preprint in perpetuity.

It is made available under a CC-BY 4.0 International license.

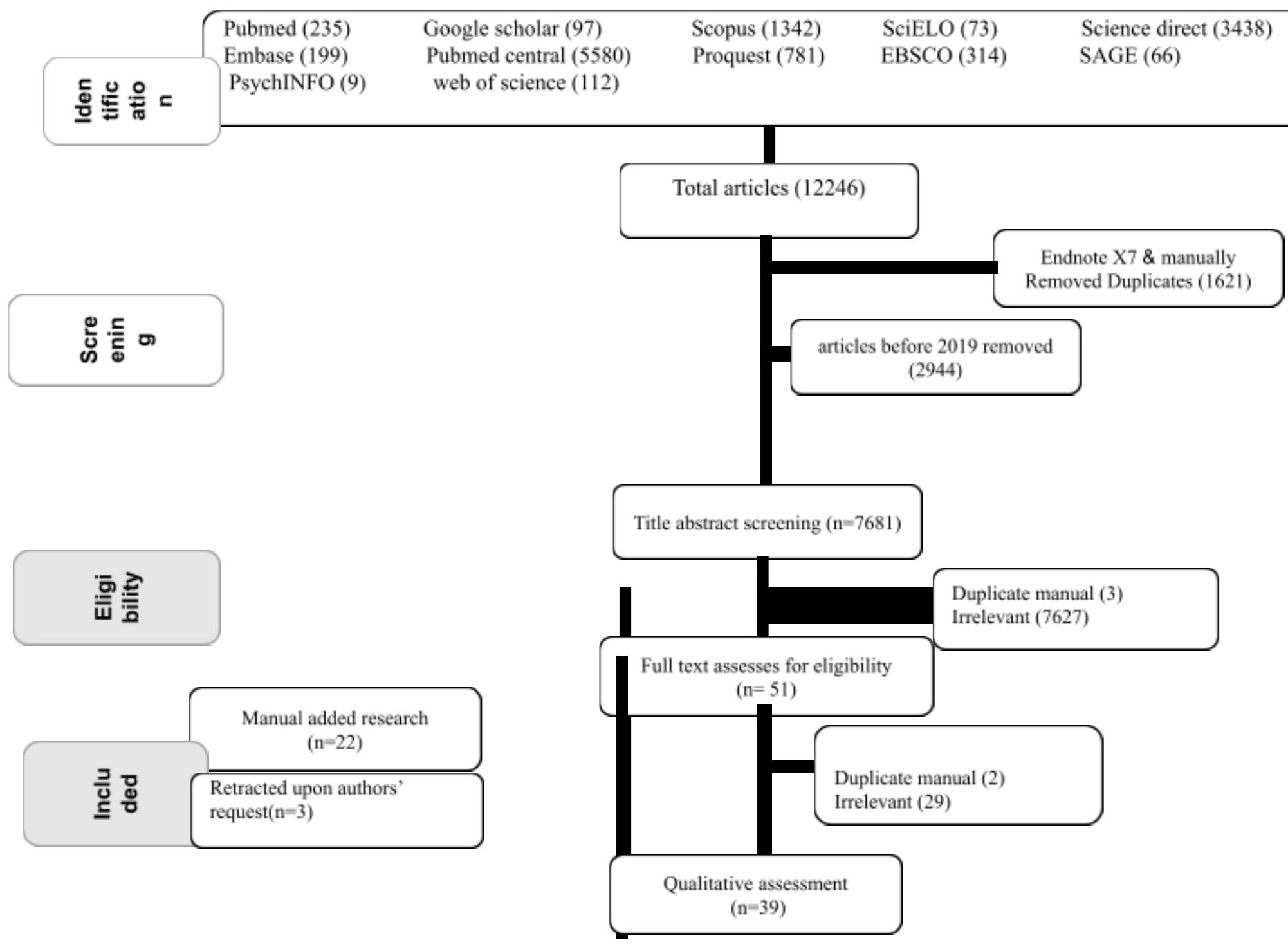

Figure 1: PRISMA flow chart of studies screened and included. 
Figure 2 presents the funnel plot of 38 studies reporting the COVID-19 vaccine hesitancy and Eggers' test $[\mathrm{t}=-1.215, \mathrm{P}$-value $=0.232]$, show absence of asymmetry and disapprove any publication bias. Figure 3 depicts the studies reporting COVID-19 vaccine acceptance and Eggers' test $[\mathrm{t}=-0.64, \mathrm{p}$-value $=0.526]$.

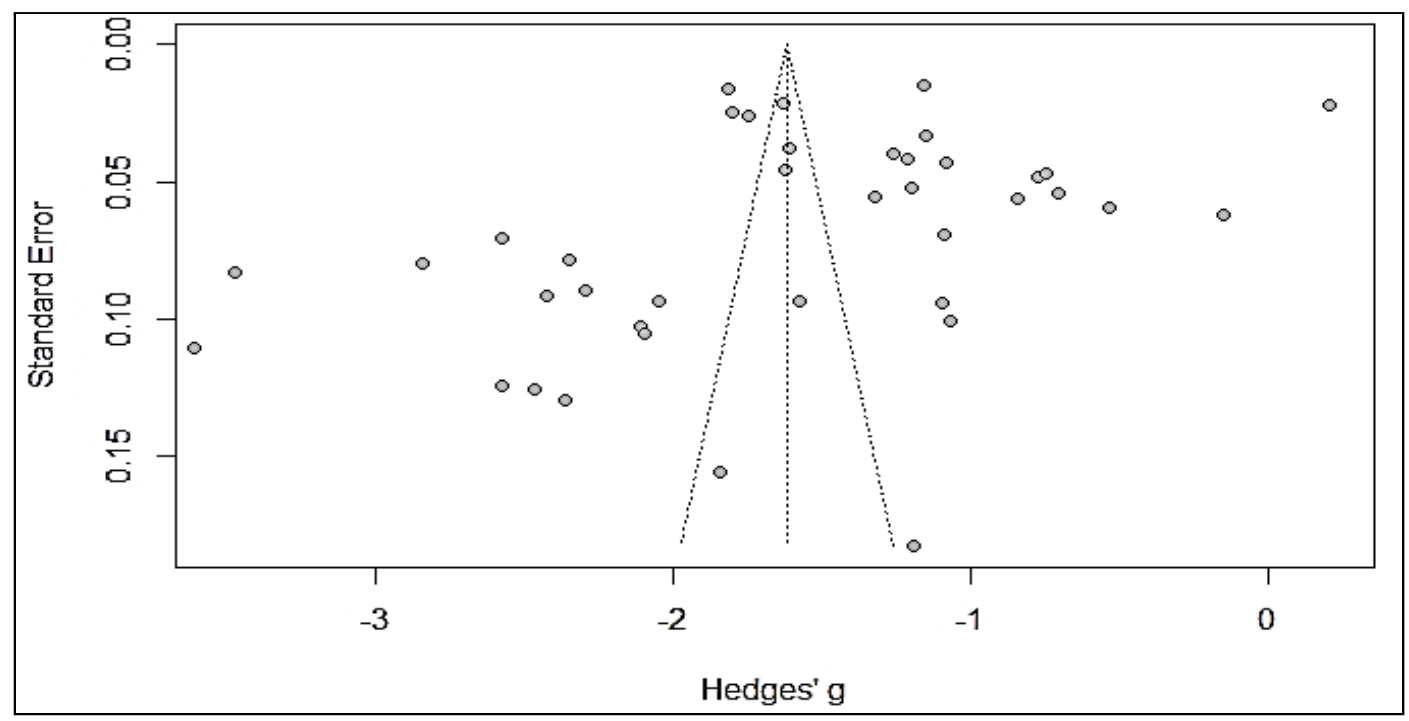

Figure 2: Funnel plot of studies reporting the COVID vaccine hesitancy

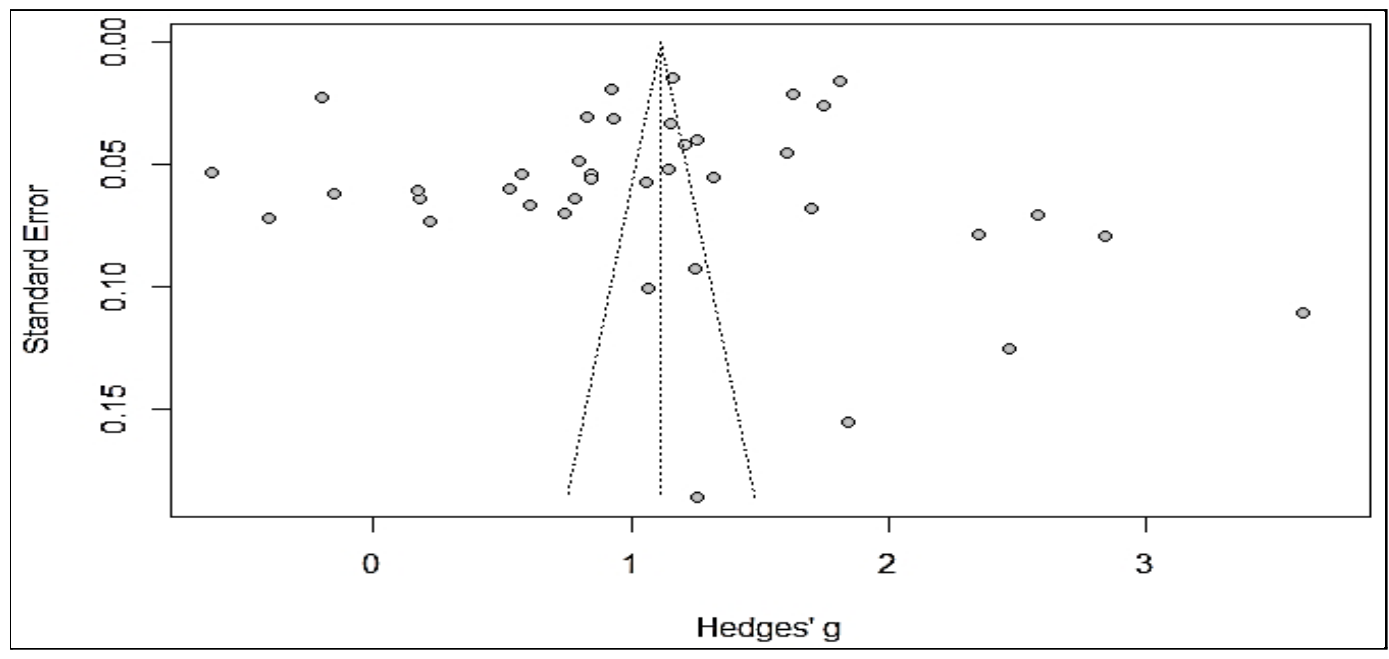

Figure 3: Funnel plot of studies reporting the COVID-19 vaccine acceptance 
medRxiv preprint doi: https://doi.org/10.1101/2021.05.15.21257261; this version posted May 18, 2021. The copyright holder for this preprint (which was not certified by peer review) is the author/funder, who has granted medRxiv a license to display the preprint in perpetuity.

It is made available under a CC-BY 4.0 International license .

Table 1 shows the main findings of included studies; all the studies were cross-sectional surveys. The total sample size was 173213 ranging from 351 in the study of Sharun et al, 2020 (18) reaching 32361 in the study of Paul et al, 2021 (19). The highest presentation of female sex was in the study of Kowk, 2021 (20) followed by Wang 2020 (21) while the lowest proportion of females was in the study of Malik et al 2020 (22). Age range was 15->85 in the study of Taylor 2020, the mean age of the study participants was the highest in the study of Taylor 2020 (23) (53 years old) and lowest in the study of Al-Mohaithef (24) 31.5 years old. Tools used to assess vaccine hesitancy were online questionnaires either Google forms or Qualtrics forms. Data was collected either through face-to-face interview, online, or both. The quality score of the studies were either very good in 5 studies, good in 20 studies, satisfactory in 12 studies, and unsatisfactory in 5 studies. The main identified predictors of vaccine hesitancy were age, gender, general trust and unknown side effects of the vaccine. The highest vaccine hesitancy were in Wang et al, 2020 study (21) (47.8\%) and Unroe et al, 2020 study (25) (45.1\%), while Murphy 2021 et al, 2020 study (26) (6\%) and Salali and Uysal,, 2020 study (27) (3\%) showed the lowest vaccine hesitancy rates.

Table 1: Summary Table of included studies in the meta-analysis

\begin{tabular}{|c|c|c|c|c|c|c|}
\hline $\begin{array}{l}\text { Author, Year, } \\
\text { Country }\end{array}$ & $\begin{array}{l}\text { Population } \\
\text { criteria }+ \\
\text { inclusion } \\
\quad \text { and } \\
\text { exclusion }\end{array}$ & $\begin{array}{l}\text { Sample size/ } \\
\text { Sex/Age }\end{array}$ & $\begin{array}{l}\text { Tool used in } \\
\text { hesitancy } \% \\
\text { estimation }\end{array}$ & Predictors & Duration & $\begin{array}{c}\text { Qualit } \\
\mathbf{y}\end{array}$ \\
\hline $\begin{array}{c}\text { Taylor, 2020, } \\
\text { Canada and USA } \\
(23)\end{array}$ & $\begin{array}{l}\text { Adults who have } \\
\text { agreed to be } \\
\text { contacted in order } \\
\text { to respond to } \\
\text { surveys. } \\
\text { Excluding } \\
\text { careless or } \\
\text { incomplete } \\
\text { responses }\end{array}$ & $\begin{array}{c}\text { Sample size } \\
=3,674 \\
(\mathrm{USA}=1,772 \\
\text { Canada }=1,902) \\
\text { Sex }=57 \% \text { male } \\
\text { Age }=53 \pm 15\end{array}$ & $\begin{array}{c}\text { Internet-based } \\
\text { self-report survey } \\
\text { delivered in English }\end{array}$ & $\begin{array}{c}\text { 1- Mistrust of vaccine benefit. } \\
\text { 2-Worry about unforeseen future negative } \\
\text { effects. } \\
\text { 3- Concerns about commercial } \\
\text { profiteering. } \\
\text { 4-Preference for natural immunity }\end{array}$ & May 6-19, 2020 & good \\
\hline $\begin{array}{c}\text { Fisher, 2020, } \\
\text { Worcester County, } \\
\text { Massachusetts, } \\
\text { United States (28) }\end{array}$ & $\begin{array}{l}\text { adults residing in } \\
\text { the United States } \\
\text { excluded } \\
\text { participants who } \\
\text { did not respond to } \\
\text { the question on } \\
\text { intent to be } \\
\text { vaccinated }\end{array}$ & $\begin{array}{c}\begin{array}{c}\text { Sample size } \\
=\end{array} \\
\text { Sex }=48.5 \% \text { male } \\
\text { Age }=48 \pm 18.1\end{array}$ & $\begin{array}{l}\text { 1-Receive an initial } \\
\text { invitation via email, } \\
\text { SMS, or phone. } \\
\text { 2-Households without } \\
\text { Internet access are } \\
\text { included and complete } \\
\text { the survey via } \\
\text { smartphone or } \\
\text { telephone interview }\end{array}$ & $\begin{array}{l}\text { Age group,race,gender,education,setting, } \\
\text { guessing as getting the coronavirus within } \\
\text { the next } 6 \text { months, influenza vaccine }\end{array}$ & $\begin{array}{c}16-20 \text { April } \\
2020\end{array}$ & good \\
\hline $\begin{array}{l}\text { La Vecchia, 2020, } \\
\text { Italy (29) }\end{array}$ & $\begin{array}{l}\text { A nationally } \\
\text { representative } \\
\text { survey of the }\end{array}$ & $\begin{array}{l}\text { Sample size } \\
\quad=1055\end{array}$ & $\begin{array}{l}\text { Using computer } \\
\text { assisted web } \\
\text { interviews (CAWI). }\end{array}$ & ---- & $\begin{array}{c}\text { September } \\
16-28,2020\end{array}$ & $\begin{array}{c}\text { satisfact } \\
\text { ory }\end{array}$ \\
\hline
\end{tabular}


medRxiv preprint doi: https://doi.org/10.1101/2021.05.15.21257261; this version posted May 18, 2021. The copyright holder for this preprint

(which was not certified by peer review) is the author/funder, who has granted medRxiv a license to display the preprint in perpetuity.

It is made available under a CC-BY 4.0 International license.

\begin{tabular}{|c|c|c|c|c|c|c|}
\hline & $\begin{array}{l}\text { general Italian } \\
\text { population }\end{array}$ & $\begin{aligned} \begin{array}{c}\text { Sex } \\
=\end{array} & 48.24 \% \\
& \text { male } \\
\text { Age } & =(15-85)\end{aligned}$ & & & & \\
\hline $\begin{array}{c}\text { Sherman , 2020, } \\
\text { United Kingdom } \\
\text { (30) }\end{array}$ & $\begin{array}{l}\text { participants were } \\
\text { broadly } \\
\text { representative of } \\
\text { the UK population } \\
\text { aged } 18 \text { years or } \\
\text { over }\end{array}$ & $\begin{array}{c}\text { Sample size } \\
=1500 \\
\text { Sex }=48.6 \% \text { male } \\
\text { Age }=46 \pm 15.8\end{array}$ & online survey & $\begin{array}{l}\text { Age,sex,religion,ethinity,qualification, } \\
\text { religion, employment status,key } \\
\text { worker,extremely clinical vulnerable, } \\
\text { previous influenza vaccination,general } \\
\text { vaccination beliefs and attitude, beliefs and } \\
\text { attitude towards COVID19,perceived Risk } \\
\text { of COVID } 19 \text {, perceived Risk of COVID } \\
19 \text { to oneself, having OR not COVID } 19 .\end{array}$ & $\begin{array}{l}\text { between 14th } \\
\text { and 17th July } \\
2020\end{array}$ & $\begin{array}{l}\text { very } \\
\text { good }\end{array}$ \\
\hline $\begin{array}{c}\text { Lucia, } 2020 \\
\text { Southeast Michigan } \\
\text { (31) }\end{array}$ & $\begin{array}{l}\text { medical students } \\
\text { aged } 18 \text { years or } \\
\text { over }\end{array}$ & $\begin{array}{c}\text { Sample size } \\
=168 \\
((168 \text { of } 494 \\
\text { medical students } \\
\text { (response rate }= \\
34 \%)) \\
\text { Sex }=43 \% \text { male }\end{array}$ & online survey & --- & $\begin{array}{l}\text { A lack of trust } \\
\text { and } \\
\text { misinformation }\end{array}$ & $\begin{array}{c}\text { satisfact } \\
\text { ory }\end{array}$ \\
\hline $\begin{array}{c}\text { Salali and Uysal, } \\
2020 \\
\text { UK and Turkey (27) }\end{array}$ & $\begin{array}{l}\text { All participants } \\
\text { were above } 18, \\
\text { residing either in } \\
\text { the UK or } \\
\text { Turkey. }\end{array}$ & $\begin{array}{c}\text { UK }(\mathrm{n}=1088) \\
\text { and Turkey }(\mathrm{n}= \\
3936)\end{array}$ & online survey & $\begin{array}{l}\text { COVID-19 vaccine acceptance, believing } \\
\text { in the natural origin of the virus }\end{array}$ & $\begin{array}{l}\text { throughout May } \\
2020\end{array}$ & $\begin{array}{l}\text { very } \\
\text { good }\end{array}$ \\
\hline $\begin{array}{l}\text { Al-Mohaithef,2020, } \\
\text { Saudi Arabia (24) }\end{array}$ & $\begin{array}{l}\text { All participants } \\
\text { were above } 18 .\end{array}$ & $\begin{array}{l}\begin{array}{c}\text { Saudi Arabia } \\
(\mathrm{n}=992)\end{array} \\
\text { Age }=(18-45) \\
\begin{array}{c}\text { Sex }= \\
\quad 34.17 \% \\
\text { male }\end{array}\end{array}$ & online survey & $\begin{array}{c}\text { Sociodemographic predictors (age } \\
\text {,gender,marital status,nationality,residence, } \\
\text { occupation,education),risk and trust to } \\
\text { health system }\end{array}$ & $\begin{array}{l}---- \\
\end{array}$ & good \\
\hline $\begin{array}{l}\text { Kreps,2020, } \\
\text { USA (32) }\end{array}$ & US adults & $\begin{array}{c}\text { USA } \\
\text { (No=2000 while } \\
\text { only } 1971 \text { were } \\
\text { respondents) } \\
\text { completed the full } \\
\text { questionnaire } \\
\text { Median Age }= \\
43(30-58) \\
\text { Sex }=49 \% \text { male }\end{array}$ & online survey & $\begin{array}{l}\text { vaccine efficacy, adverse effects, and } \\
\text { protection duration) and political factors } \\
\text { (eg, US Food and Drug Administration } \\
\text { approval process, national origin of } \\
\text { vaccine, and endorsements), Health care } \\
\text { attitudes and practices, political } \\
\text { partisanship, and demographic } \\
\text { characteristics, including age, sex, and } \\
\text { race/ethnicity }\end{array}$ & July $9,2020$. & good \\
\hline $\begin{array}{c}\text { Gagneux-Brunon, } \\
2020, \\
\text { France }(33)\end{array}$ & $\begin{array}{c}\text { General } \\
\text { Population and } \\
\text { Health Care } \\
\text { Workers in France }\end{array}$ & $\begin{array}{l}\text { France }(\mathrm{n}=2047) \\
\text { Age }=(<30->65) \\
\text { Sex }=26 \% \text { male }\end{array}$ & $\begin{array}{l}\text { Combined (Online } \\
\text { Survey + written } \\
\text { questionnaire ) }\end{array}$ & $\begin{array}{c}\text { Age,gender,professions,chronic medical } \\
\text { conditions, getting Flu vaccine during the } \\
\text { previous season, Fear about COVID-19, } \\
\text { Perceived individual risk, Vaccine } \\
\text { hesitancy }\end{array}$ & $\begin{array}{l}\text { From 26th } \\
\text { March to 2nd } \\
\text { July } 2020 .\end{array}$ & $\begin{array}{l}\text { unsatisf } \\
\text { actory }\end{array}$ \\
\hline $\begin{array}{l}\text { Lin, 2020, } \\
\text { China (34) }\end{array}$ & & $\begin{array}{c}3,541 \\
\text { higher } \\
\text { representation of } \\
\text { participants aged } \\
26 \text { to } 35 \text { years old } \\
(47.2 \%) \\
\mathrm{M}=48.1 \% \\
\mathrm{~F}=51.9 \% \\
\end{array}$ & Online questionnaire & - - - & 1-19 May 2020 & good \\
\hline $\begin{array}{c}\text { Barello, } \\
\text { Serena,2020, } \\
\text { Italy (35) } \\
\end{array}$ & $\begin{array}{c}\text { Italian University } \\
\text { students }\end{array}$ & 934 & Online survey & NA & $\begin{array}{l}\text { 1st-19th May } \\
2020\end{array}$ & $\begin{array}{l}\text { unsatisf } \\
\text { actory }\end{array}$ \\
\hline $\begin{array}{l}\text { Dror, 2020, Israel } \\
\text { (36) }\end{array}$ & NA & 1941 & $\begin{array}{l}\text { Qualtrics online } \\
\text { survey }\end{array}$ & NA & 2-weeks & good \\
\hline
\end{tabular}


medRxiv preprint doi: https://doi.org/10.1101/2021.05.15.21257261; this version posted May 18, 2021. The copyright holder for this preprint (which was not certified by peer review) is the author/funder, who has granted medRxiv a license to display the preprint in perpetuity.

It is made available under a CC-BY 4.0 International license .

\begin{tabular}{|c|c|c|c|c|c|c|}
\hline $\begin{array}{c}\text { Akarsu, 2020, } \\
\text { Turkey (37) }\end{array}$ & $\begin{array}{l}\text { Social Media and } \\
\text { smartphone users }\end{array}$ & \begin{tabular}{ll}
\multicolumn{2}{c}{852} \\
Female & $62.8 \%$ \\
Male & $37.2 \%$
\end{tabular} & Online Survey & & & good \\
\hline $\begin{array}{c}\text { Freeman, 2021, } \\
\text { UK (38) }\end{array}$ & & $\begin{array}{l}5,114 \text { UK adults } \\
\text { Age mean } \\
\text { (SD)=46.9 }(17.1) \\
\text { male; female; } \\
\text { non-binary; prefer } \\
\text { not say }=2574 ; \\
2515 ; 20 ; 5 \\
\end{array}$ & Online Survey & $\begin{array}{c}\text { lower age, female gender, lower education, } \\
\text { lower income, black and mixed ethnicities, } \\
\text { not being single or widowed, not being a } \\
\text { homeowner, not being employed full-time, } \\
\text { not retired, a change in working, } \\
\text { having a child at school. }\end{array}$ & $\begin{array}{c}\text { 24th } \\
\text { September-17th } \\
\text { October } 2020\end{array}$ & good \\
\hline $\begin{array}{l}\text { Butter,2020, } \\
\text { UK (39) }\end{array}$ & $\begin{array}{l}\text { UK adults who } \\
\text { took part in the } \\
\text { 1-month } \\
\text { follow-up } \\
\text { survey of the } \\
\text { COVID19 } \\
\text { Psychological } \\
\text { Wellbeing Study. } \\
\text { AND } \\
\text { only individuals } \\
\text { who } \\
\text { reported not } \\
\text { having been } \\
\text { previously } \\
\text { diagnosed with } \\
\text { COVID-19 } \\
\text { (formally } \\
\text { diagnosed, } \\
\text { diagnosed by GP } \\
\text { or self-diagnosed) }\end{array}$ & $\begin{array}{c}\mathbf{1 6 0 5} \\
\text { Male } \\
\text { Key workers } \\
146(25.0) \\
\text { Non-Key } \\
\text { workers 347 } \\
(34.3) \\
\text { Female } \\
\text { Key workers 437 } \\
\text { (75.0) } \\
\text { Non-Key } \\
\text { workers 664 } \\
(65.7) \\
\mathbf{A g e} \\
\mathbf{1 8 - 2 4} \\
\text { Key workers } 60 \\
\text { (10.3) } \\
\text { Non-Key workers } \\
211(20.7) \\
\mathbf{2 5 - 3 4} \\
\text { Key workers } \\
197(33.7) \\
\text { Non-Key workers } \\
320(31.3) \\
\mathbf{3 5 - 4 4} \\
\text { Key workers } \\
175(30.0) \\
\text { Non-Key workers } \\
210(20.6) \\
\mathbf{4 5 - 5 4} \\
\text { Key workers } \\
100(17.1) \\
\text { Non-Key workers } \\
136(13.3) \\
\mathbf{5 5 +} \\
\text { Key workers } 52 \\
(8.9) \\
\text { Non-Key workers } \\
144(14.1) \\
\end{array}$ & online survey & $\begin{array}{c}\text { Female1.96 }(1.16-3.32) \\
\text { Age group 25-342.41 }(1.48-3.94) \\
\text { Age group 35-441.96 }(1.12-3.45) \\
\text { Age group 45-542.91 }(1.62-5.24)\end{array}$ & $\begin{array}{l}\text { April and 18th } \\
\text { May 2020. } \\
\text { between } 2 \text { d } \\
\end{array}$ & $\begin{array}{c}\text { satisfact } \\
\text { ory }\end{array}$ \\
\hline $\begin{array}{c}\text { Muqattash,2020, } \\
\text { UAE (40) }\end{array}$ & $\begin{array}{l}\text { (aged } 18 \text { and } \\
\text { above) living in } \\
\text { the UAE }\end{array}$ & $\begin{array}{c}1109 \\
\mathrm{M}=27.86 \% \\
\mathrm{~F}=72.14 \% \\
\text { 1-[18 to } 25] 143 \\
12.89 \% \\
\text { 2- }[26 \text { to } 35] \\
310 \\
27.95 \% \\
\text { 3- }[36 \text { to } 45] \\
437 \\
39.40 \% \\
\text { 4- }[45 \text { and over[ } \\
219 \\
19.75 \% \\
\end{array}$ & $\begin{array}{l}\text { Google Forms } \\
\text { platform survey }\end{array}$ & & $\begin{array}{c}\text { July 4th to } \\
\text { August 4th } 2020\end{array}$ & $\begin{array}{c}\text { satisfact } \\
\text { ory }\end{array}$ \\
\hline $\begin{array}{l}\text { Ward, 2020, } \\
\text { France (41) }\end{array}$ & Age $\geq 18$ & 5018 & Online survey & $\begin{array}{c}\text { male } 0.69[0.59 ; 0.82] \\
\quad \underline{\text { Gender }} \\
<35 \text { y.o } 1.36[1.14 ; 1.62] \\
>64 \text { y.o } 0.29[0.22 ; 0.38] \\
\text { COVID-19-related concern } \\
\end{array}$ & $\begin{array}{c}4 \text { weeks April } \\
2020\end{array}$ & good \\
\hline
\end{tabular}


medRxiv preprint doi: https://doi.org/10.1101/2021.05.15.21257261; this version posted May 18, 2021. The copyright holder for this preprint (which was not certified by peer review) is the author/funder, who has granted medRxiv a license to display the preprint in perpetuity.

It is made available under a CC-BY 4.0 International license.

\begin{tabular}{|c|c|c|c|c|c|c|}
\hline & & & & $\begin{array}{c}\text { High }(>8) 0.68[0.55 ; 0.84] \\
\text { Partisan preference } \\
\text { Far-Left parties } 1.43[1.07 ; 1.91] \\
\text { Left/Center/Right } 1.47[1.12 ; 1.92] \\
\text { No preference and abstained in } 20171.74 \\
{[1.26 ; 2.41]}\end{array}$ & & \\
\hline $\begin{array}{l}\text { Unroe, 2020, } \\
\text { India (25) }\end{array}$ & $\begin{array}{c}\text { Nursing home and } \\
\text { assisted living } \\
\text { facility staff }\end{array}$ & $\begin{array}{c}8,243 \\
\mathrm{~F}=87.2 \% \\
\mathrm{M}=12.8 \% \\
\end{array}$ & $\begin{array}{c}\text { Survey via text } \\
\text { message or email }\end{array}$ & Side effects & $\begin{array}{l}\text { November } 14 \\
\text { and } 17,2020\end{array}$ & $\begin{array}{c}\text { satisfact } \\
\text { ory }\end{array}$ \\
\hline $\begin{array}{l}\text { Wang,.K, 2020, } \\
\text { china (21) }\end{array}$ & $\begin{array}{c}\text { Nurses, } \\
\text { administrative or } \\
\text { academic } \\
\text { positions excluded }\end{array}$ & $\begin{array}{c}806 \\
\mathrm{~F}=87.5 \% \\
\mathrm{M}=(12.5) \% \\
21.6 \% \text { nurses } \\
\text { aged } 18-29, \\
31.1 \% \text { aged } \\
30-39,27.1 \% \\
\text { aged } 40-49 \text { and } \\
20.2 \% \text { aged } 50 \text { or } \\
\text { above }\end{array}$ & Online survey & $\begin{array}{c}\text { Gender2.78(1.69, 4.58) } \\
\text { Having chronic conditions } 1.83(1.22,2.77) \\
\text { Public or private1.67(1.11, 2.51) }\end{array}$ & $\begin{array}{l}26 \text { February and } \\
31 \text { March } 2020\end{array}$ & $\begin{array}{c}\text { unsatisf } \\
\text { actory }\end{array}$ \\
\hline $\begin{array}{l}\text { Goldman, 2020, } \\
\text { (USA, Canada, } \\
\text { Israel, Japan, Spain, } \\
\text { and Switzerland) } \\
\quad(42)\end{array}$ & Caregiver families & $\begin{array}{c}1541 \\
\mathrm{~F}=72 \% \\
\mathrm{M}=25.5 \% \\
\text { Age } \\
\text { mean=39.9(SD7.6 } \\
\end{array}$ & Online survey & & $\begin{array}{l}\text { 26thMarch-31st } \\
\text { May } 2020\end{array}$ & good \\
\hline $\begin{array}{l}\text { Reiter, 2020, } \\
\text { USA (43) }\end{array}$ & Adults & $\begin{array}{c}2006 \\
\mathrm{~F}=56 \% \\
\mathrm{M}=43 \% \\
\end{array}$ & Online survey & & May 2020 & good \\
\hline $\begin{array}{c}\text { Wang J, 2020, } \\
\text { China (44) }\end{array}$ & Adults & $\begin{array}{c}2058 \\
\mathrm{~F}=54.2 \% \\
\mathrm{M}=45.8\end{array}$ & Online survey & NA & 更 & good \\
\hline $\begin{array}{l}\text { Sharun , 2020, } \\
\text { India (18) }\end{array}$ & Adults & $\begin{array}{c}351 \\
\mathrm{~F}=58.1 \% \\
\mathrm{M}=41.3 \%\end{array}$ & Online survey & $\overline{\mathrm{NA}}$ & October 2020 & $\begin{array}{l}\text { very } \\
\text { good }\end{array}$ \\
\hline $\begin{array}{l}\text { Lazarus , 2020, } \\
\text { Global (11) }\end{array}$ & Adults & $\begin{array}{c}13,426 \\
\mathrm{~F}=53.5 \% \\
\mathrm{M}=46.5 \&\end{array}$ & Online survey & NA & NA & good \\
\hline $\begin{array}{l}\text { Kose, 2020, } \\
\text { Turkey (45) }\end{array}$ & $\begin{array}{l}\text { healthcare } \\
\text { personnel }\end{array}$ & $\begin{array}{c}1138 \\
\mathrm{~F}=72.5 \% \\
\mathrm{M}=27.5 \%\end{array}$ & $\begin{array}{l}\text { Google Forms } \\
\text { questionnaire }\end{array}$ & $\begin{array}{c}\text { gender } \\
\text { Age group } \\
\text { Occupation } \\
\text { Flu-vaccination status }\end{array}$ & $\begin{array}{c}\text { 17th -20th } \\
\text { September } 2020\end{array}$ & $\begin{array}{c}\text { satisfact } \\
\text { ory }\end{array}$ \\
\hline $\begin{array}{l}\text { Biasio , 2020, } \\
\text { Italy (46) }\end{array}$ & Adults & $\begin{array}{c}885 \\
\text { Males }(49.9 \%) \\
\text { Females }(50.1 \%) \\
\end{array}$ & Online survey & NA & 2 weeks & good \\
\hline $\begin{array}{l}\text { Grüner., 2020, } \\
\text { Germany (47) }\end{array}$ & $\begin{array}{c}\text {-university } \\
\text { students who are } \\
\text { enrolled in } \\
\text { medicine or a } \\
\text { healthcare degree } \\
\text { programme } \\
\text { (Healthcare } \\
\text { students) } \\
\text {-university } \\
\text { students who are } \\
\text { not } \\
\text { (Non-healthcare } \\
\text { students) }\end{array}$ & 2,077 & Online survey & NA & $\begin{array}{c}18.5 .2020-2.8 .20 \\
20\end{array}$ & $\begin{array}{c}\text { satisfact } \\
\text { ory }\end{array}$ \\
\hline $\begin{array}{l}\text { Malik, 2020, } \\
\text { USA (22) }\end{array}$ & Adults & $\begin{array}{c}672 \\
\text { Males ( } 72 \%) \\
\text { compared to }\end{array}$ & $\begin{array}{l}\text { Qualtrics Online } \\
\text { survey }\end{array}$ & & May 2020 & good \\
\hline
\end{tabular}


medRxiv preprint doi: https://doi.org/10.1101/2021.05.15.21257261; this version posted May 18, 2021. The copyright holder for this preprint

(which was not certified by peer review) is the author/funder, who has granted medRxiv a license to display the preprint in perpetuity.

It is made available under a CC-BY 4.0 International license .

\begin{tabular}{|c|c|c|c|c|c|c|}
\hline & & $\begin{array}{c}\text { females, older } \\
\text { adults }(\geq 55 \text { years; } \\
78 \%) \text { compared to } \\
\text { younger adults } \\
\end{array}$ & & & & \\
\hline $\begin{array}{l}\text { Paul, 2020, } \\
\text { UK (19) }\end{array}$ & $\begin{array}{c}\text { Adults who had } \\
\text { started the vaccine } \\
\text { module } \\
\text { administered from } \\
7 \text { September to } 5 \\
\text { October } 2020 .\end{array}$ & $\begin{array}{c}32,361 \\
\text { participants } \\
\text { Male } 49.4 \% \\
\text { Female } 50.6 \%\end{array}$ & $\begin{array}{l}\text { Data were drawn from } \\
\text { the COVID-19 Social } \\
\text { Study online survey }\end{array}$ & $\begin{array}{c}\text { Being female RRR }=1.45 ; 95 \% \text { CI: } 1.27 \text { to } \\
1.65 \\
\text { key workers (uncertain: } \mathrm{RRR}=1.18 ; 95 \% \\
\text { CI: } 1.01 \text { to } 1.38 \text { ) } \\
\text { People living with children (uncertain: } \\
\text { RRR }=1.38 ; 95 \% \text { CI: } 1.13 \text { to } 1.70 \\
\text { Socio-economic factors } \\
\text { levels of education }\end{array}$ & $\begin{array}{c}\text { Started on March } \\
2020 \\
\text { Duration NA }\end{array}$ & $\begin{array}{c}\text { satisfact } \\
\text { ory }\end{array}$ \\
\hline $\begin{array}{c}\text { Kwok, 2020, } \\
\text { Hong Kong }(20)\end{array}$ & & $\begin{array}{c}1,205 \text { eligible } \\
\text { nurses (mean } \\
\text { age }=40.79, S D= \\
10.47 ; 90 \% \text { being } \\
\text { female) }\end{array}$ & online survey & $\begin{array}{c}\text { 1-Confidence } \\
\text { 2-Complacency } \\
\text { 3-Collective responsibility }\end{array}$ & $\begin{array}{l}\text { mid-March and } \\
\text { late April } 2020\end{array}$ & good \\
\hline $\begin{array}{c}\text { Edwards,2020, } \\
\text { Australia (48) }\end{array}$ & Adults & $\begin{array}{c}\text { 3,061 adults } \\
\text { Age 18-75 }\end{array}$ & online survey & $\begin{array}{c}\text { Age } \\
\text { Sex } \\
\text { university degree } \\
\text { neighbourhood differences } \\
\text { household income } \\
\text { who downloaded the COVID-Safe App } \\
\text { who thought too much fuss } \\
\text { who voted for the Coalition } \\
\text { who voted for Labor } \\
\text { confidence in their state or territory } \\
\text { government or in their hospitals and health } \\
\text { system } \\
\text { religion } \\
\text { populist views } \\
\text { who were more likely to support migration } \\
\text { levels of social distancing }\end{array}$ & & good \\
\hline $\begin{array}{l}\text { Detoc,2020, } \\
\text { France (49) }\end{array}$ & & $\begin{array}{c}3259 \\
\mathrm{~F}=67.4 \% \\
\mathrm{M}=32.6\end{array}$ & online survey & NA & $\begin{array}{l}\text { 26th march-20th } \\
\text { April } 2020\end{array}$ & $\begin{array}{c}\text { satisfact } \\
\text { ory }\end{array}$ \\
\hline $\begin{array}{l}\text { Adebesie, 2020, } \\
\text { Nigeria (50) }\end{array}$ & & $\begin{array}{c}517 \\
\mathrm{~F}=43.1 \% \\
\mathrm{M}=56.9 \% \\
\mathrm{Age} \geq 15\end{array}$ & online survey & $\begin{array}{c}\text { Age } \\
\text { employment } \\
\text { education level }\end{array}$ & & good \\
\hline $\begin{array}{c}\text { Murphy, 2021, } \\
\text { UK (26) }\end{array}$ & NA & $\begin{array}{c}2025 \\
\mathrm{~F}=51.7 \\
\mathrm{M}=48.3 \%\end{array}$ & online survey & $\begin{array}{c}\text { Gender } \\
\text { Age group }\end{array}$ & NA & $\begin{array}{c}\text { satisfact } \\
\text { ory }\end{array}$ \\
\hline $\begin{array}{l}\text { Murphy 2021, } \\
\text { Ireland (26) }\end{array}$ & $\mathrm{NA}$ & $\begin{array}{c}1041 \\
\mathrm{~F}=51.5 \% \\
\mathrm{M}=48.2 \%\end{array}$ & online survey & $\begin{array}{c}\text { Gender } \\
\text { Age group } \\
\text { Mental health history }\end{array}$ & NA & $\begin{array}{c}\text { satisfact } \\
\text { ory }\end{array}$ \\
\hline $\begin{array}{c}\text { Barry,2020, } \\
\text { Saudi-Arabia (51) }\end{array}$ & $\begin{array}{l}\text { Healthcare } \\
\text { workers }\end{array}$ & $\begin{array}{c}1058 \\
\mathrm{~F}=62.4 \% \\
\mathrm{M}=37.6 \%\end{array}$ & online survey & $\begin{array}{c}\text { Efficient data } \\
\text { Lack of sufficient safety } \\
\text { Potential adverse effects } \\
\text { Belief that vaccine would be ineffective } \\
\text { Complacency } \\
\text { confidence } \\
\end{array}$ & $\begin{array}{l}\text { 4-14 December } \\
2020\end{array}$ & $\begin{array}{l}\text { very } \\
\text { good }\end{array}$ \\
\hline $\begin{array}{l}\text { Chen,2021, } \\
\text { China (52) }\end{array}$ & & 3195 & online survey & $\begin{array}{l}\text { Lack of confidence } \\
\text { Complacency } \\
\text { Risk of the vacc. } \\
\text { Attention frequency }\end{array}$ & NA & $\begin{array}{l}\text { very } \\
\text { good }\end{array}$ \\
\hline $\begin{array}{l}\text { Meyer,2020, } \\
\text { USA (53) }\end{array}$ & $\begin{array}{l}\text { Patient-facing } \\
\text { HCWs and other } \\
\text { roles }\end{array}$ & 16158 & online survey & $\begin{array}{c}\text { Unknown risk } \\
\text { Insufficient data } \\
\text { Known side effects } \\
\text { Don't trust FDA } \\
\text { Privacy concerns about Geisinger and state } \\
\text { tracking } \\
\text { Depend on which vaccine and concerns } \\
\text { about mRNA } \\
\text { Not at high risk for serious disease } \\
\text { Not at high risk for infection } \\
\text { Had COVID or vacc. Unnecessary }\end{array}$ & $\begin{array}{c}\text { December } \\
2020 \text { (month) }\end{array}$ & $\begin{array}{c}\text { satisfact } \\
\text { ory }\end{array}$ \\
\hline Robertson,2021, & $\overline{\mathrm{NA}}$ & 12,035 & online survey & Future unknown effect & Nov.-Dec.2020 & good \\
\hline
\end{tabular}


medRxiv preprint doi: https://doi.org/10.1101/2021.05.15.21257261; this version posted May 18, 2021. The copyright holder for this preprint

(which was not certified by peer review) is the author/funder, who has granted medRxiv a license to display the preprint in perpetuity.

It is made available under a CC-BY 4.0 International license .

\begin{tabular}{|c|c|c|c|c|c|}
\hline UK (54) & & & $\begin{array}{c}\text { Lack of trust in vaccine } \\
\text { Side effects } \\
\text { Gender } \\
\text { Age } \\
\text { education }\end{array}$ & & \\
\hline $\begin{array}{c}\text { Kerr, } 2020, \\
12 \text { countries } \\
\text { (Australia, China, } \\
\text { France, Germany, } \\
\text { Italy, Japan, Korea, } \\
\text { Mexico, Spain, } \\
\text { Sweden, UK, USA) } \\
\text { (55) }\end{array}$ & 更 & $\begin{array}{c}\text { Qualtrics online } \\
\text { survey }\end{array}$ & $\begin{array}{c}\text { Demographic numeracy } \\
\text { Political ideology } \\
\text { General social trust } \\
\text { Prosociability } \\
\text { General trust in medical scientific experts } \\
\text { General trust in government } \\
\text { Specific trust in national science advisors, } \\
\text { WHO } \\
\text { Perceived likelihood of infection } \\
\text { Worry about COVID }\end{array}$ & $\begin{array}{l}\text { March - Oct. } \\
2020\end{array}$ & good \\
\hline
\end{tabular}

$\mathrm{F}=$ Female, $\mathrm{M}=$ Male, $\mathbf{N A}=$ Not Available

\section{Predictors of COVID-19 vaccine acceptance and hesitancy}

Multiple factors were associated with vaccine hesitancy Table(1). Previously receiving influenza vaccine is the main factor that determines the acceptance of COVID-19 vaccine. Individuals reporting intake of influenza vaccine were more likely to accept COVID-19 vaccine than those who did not receive it previously $(21,28,33)$.Some socio-demographic characteristics were considered to influence the acceptance of the vaccine. Being young was associated with no or not sure response towards the intake of COVID-19 vaccine $(28,38,41)$, while older individuals were more likely to accept the vaccine intake $(24,33)$. Regarding the gender, males were more likely to accept the vaccine rather than females $(21,33,38,45)$.. Low education levels and income, being not employed in a full time job or retired were associated with refusal of the vaccine $(19,28$, $38,41)$, while those with professional private work were more likely to accept the vaccine (21). The marital status also affects the response to vaccine acceptance, being single or widowed were associated with hesitancy (38), while married individuals were more likely to accept the vaccine (24). Racial and ethnic groups were noticed to affect the acceptance of vaccine. Black race and mixed ethnicity were associated with hesitancy towards the vaccine $(28,38)$. Other factors that increase the acceptance towards the vaccine is the presence of trusted health systems (24), the fear from getting infected with the virus (33) and having chronic diseases (21). While factors that increase the refusal of the vaccine involve the suspicion from its efficacy and effectiveness (21), individuals may think 
medRxiv preprint doi: https://doi.org/10.1101/2021.05.15.21257261; this version posted May 18, 2021. The copyright holder for this preprint

about the side effects and do not believe that the vaccine will work, or they trust their immune system and are not afraid of getting sick (45).

\section{Pooled proportion of COVID-19 vaccine hesitancy and acceptance}

Using the random effect model, (figure 5) the pooled proportion of COVID-19 vaccine hesitancy among 173213 participants recruited from 39 studies was 17\% (95\% CI: 14-20). Vaccine hesitancy ranged from 55\% (95\% CI: 85-87) in the study of Unroe, 2020 (25) to 3\% (95\% CI: 3-4) in the study of Salali, 2020 (27) and 3\% (95\% CI: 2-3) in Chen, 2021 (52), with high heterogeneity $\left(\mathrm{I}^{2}=100 \%\right)$. To identify the cause of such heterogeneity, researchers conducted Leave one out sensitivity analysis, sub-group analysis, or meta-regression, however, these analyses failed to explain this heterogeneity. On the other hand, the pooled proportion of COVID-19 vaccine acceptance (figure 6) was 75\% (95\%CI: 71-79). The vaccine acceptance was the highest in Chen, 2021 (97, 95\% CI =97-98) and the lowest in Goldman, $2020(35,95 \% \mathrm{CI}=32-37)$. Similar to the vaccine hesitancy, the vaccine acceptance showed high heterogeneity $\left(\mathrm{I}^{2}=100 \%\right)$. However, meta-regression revealed that case fatality, sample size, the number of reported cases per country and the type of study setting explained $38.52 \%$ of the model heterogeneity ( $\mathrm{p}$-value $<0.0001$ ), the estimated amount of residual heterogeneity $\left(T^{2}\right)$ was $0.3201(\mathrm{SE}=0.1350)$. However, only case fatality and the number of reported cases had a significant effect on vaccine acceptance. The pooled proportion of vaccine acceptance increased by $39.95 \%(95 \% \mathrm{CI}=20.1-59.8)$ for each $1 \%$ increase in case fatality $(p<0.0001)$ and decreased by $0.1 \%(95 \% \mathrm{CI}=-0.2$ to -0.01$)$ for each 1000 reported case of COVID-19 $(p=0.0183)$.Figure (4) shows the results of the meta-regression models between the case fatality (\%) and the proportion of vaccine hesitancy and vaccine acceptance, respectively by type of setting and study sample size. 
medRxiv preprint doi: https://doi.org/10.1101/2021.05.15.21257261; this version posted May 18, 2021. The copyright holder for this preprint (which was not certified by peer review) is the author/funder, who has granted medRxiv a license to display the preprint in perpetuity.

It is made available under a CC-BY 4.0 International license .

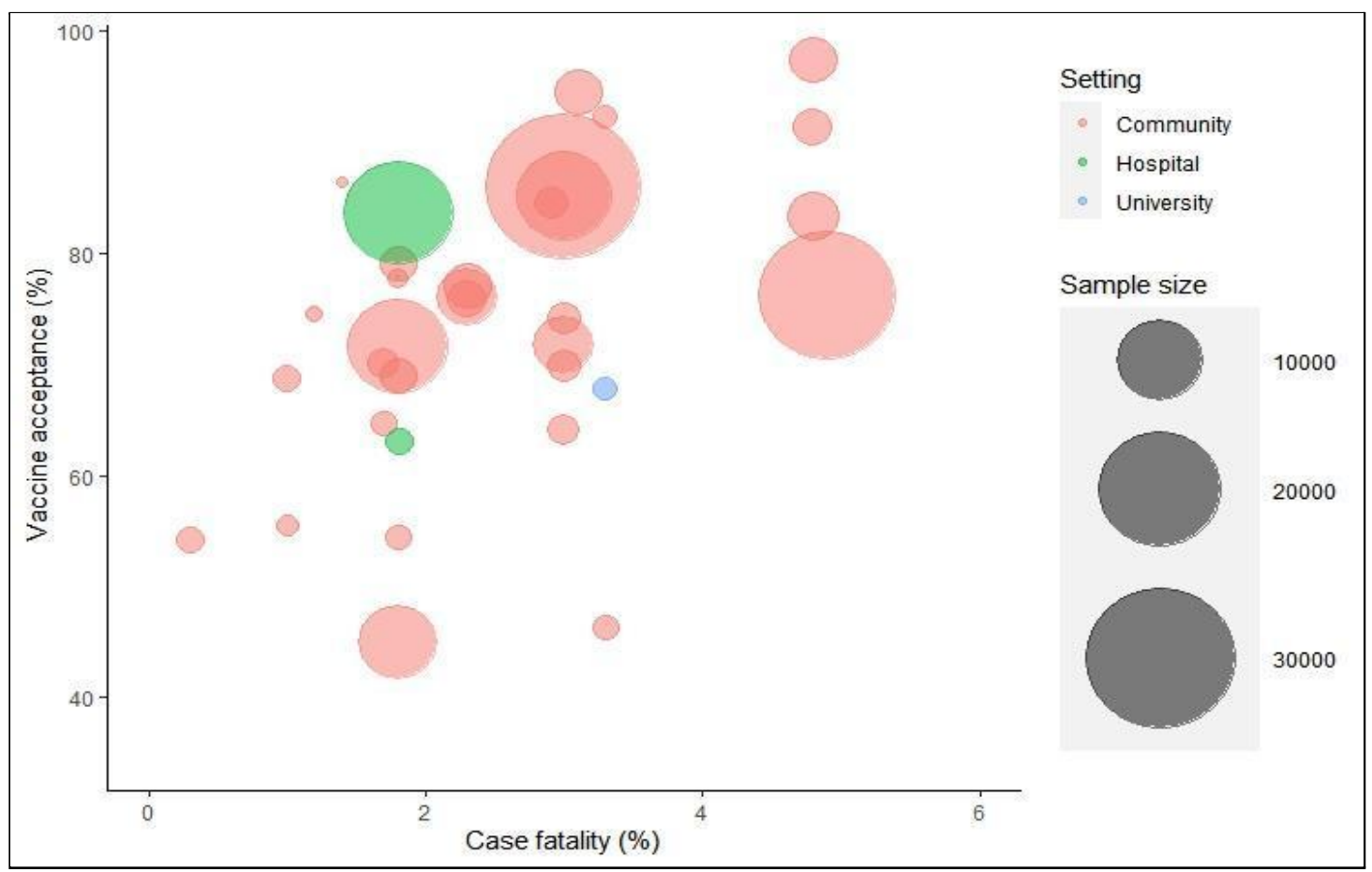

Figure (4) the relation between vaccine acceptance (\%) and case fatality (\%) by sample size and study setting 
medRxiv preprint doi: https://doi.org/10.1101/2021.05.15.21257261; this version posted May 18, 2021. The copyright holder for this preprint (which was not certified by peer review) is the author/funder, who has granted medRxiv a license to display the preprint in perpetuity.

It is made available under a CC-BY 4.0 International license

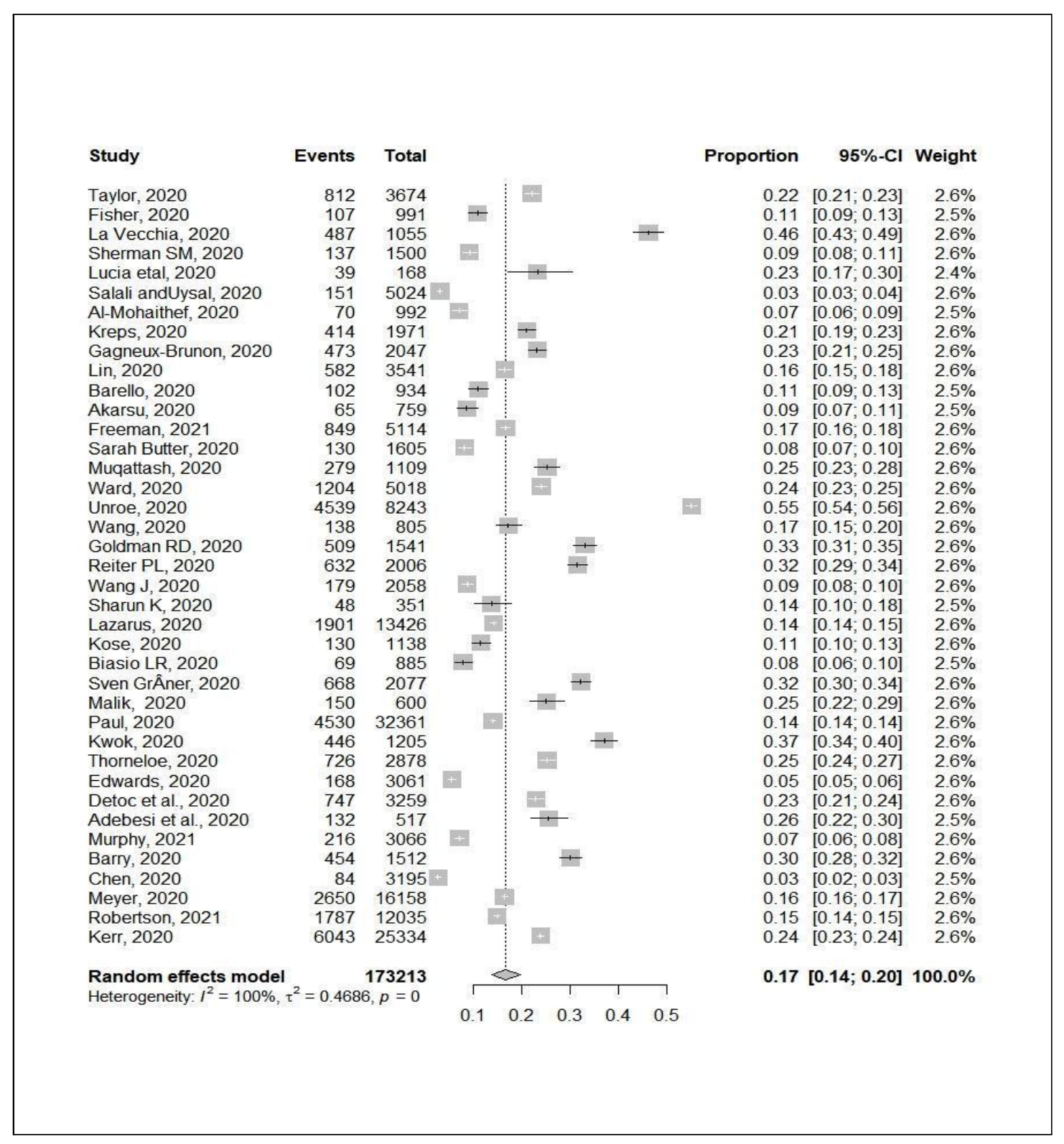

Figure (5) Forest plot of pooled prevalence of vaccine hesitancy 
medRxiv preprint doi: https://doi.org/10.1101/2021.05.15.21257261; this version posted May 18, 2021. The copyright holder for this preprint (which was not certified by peer review) is the author/funder, who has granted medRxiv a license to display the preprint in perpetuity.

It is made available under a CC-BY 4.0 International license.

Study

Taylor, 2020

Fisher, 2020

La Vecchia, 2020

Sherman SM, 2020

Lucia etal, 2020

Salali andUysal, 2020

Al-Mohaithef, 2020

Kreps, 2020

Gagneux-Brunon, 2020

Lin, 2020

Barello, 2020

Akarsu, 2020

Freeman, 2021

Sarah Butter, 2020

Muqattash, 2020

Ward, 2020

Unroe, 2020

Wang, 2020

Goldman RD, 2020

Reiter PL, 2020

Wang J, 2020

Sharun K, 2020

Lazarus, 2020

Kose, 2020

Biasio LR, 2020

Sven GrÂner, 2020

Malik, 2020

Paul, 2020

Kwok, 2020

Thorneloe, 2020

Edwards, 2020

Detoc et al., 2020

Adebesi et al., 2020

Murphy, 2021

Barry, 2020

Chen, 2020

Meyer, 2020

Robertson, 2021

Kerr, 2020

Random effects model

Heterogeneity: $I^{2}=100 \%, \tau^{2}=0.4610, p=0$

\begin{tabular}{rr} 
Events & Total \\
& \\
2862 & 3674 \\
539 & 991 \\
488 & 1055 \\
960 & 1500 \\
130 & 167 \\
3501 & 5024 \\
642 & 992 \\
1557 & 1971 \\
1554 & 2047 \\
2950 & 3541 \\
633 & 934 \\
421 & 759 \\
3667 & 5114 \\
1191 & 1605 \\
601 & 1109 \\
3814 & 5018 \\
3709 & 8243 \\
322 & 805 \\
536 & 1541 \\
1384 & 2006 \\
1879 & 2058 \\
303 & 351 \\
9604 & 13426 \\
781 & 1138 \\
816 & 885 \\
1413 & 1670 \\
522 & 672 \\
27831 & 32361 \\
759 & 1205 \\
1156 & 1654 \\
2893 & 3061 \\
2512 & 3259 \\
385 & 517 \\
2850 & 3066 \\
1058 & 1512 \\
3111 & 3195 \\
13508 & 16158 \\
10248 & 12035 \\
19291 & 25334 \\
& \\
\hline 0.4610 & 171653 \\
& $p$ \\
& \\
\hline
\end{tabular}

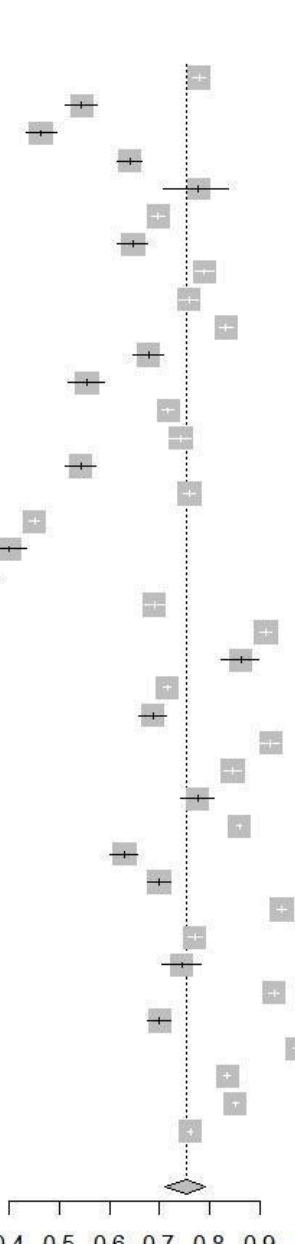

Proportion $\quad 95 \%-\mathrm{Cl}$ Weight

$0.78[0.77 ; 0.79]-2.6 \%$

$0.54[0.51 ; 0.58] \quad 2.6 \%$

$0.46[0.43 ; 0.49] \quad 2.6 \%$

$0.64[0.62 ; 0.66] \quad 2.6 \%$

$0.78[0.71 ; 0.84] \quad 2.4 \%$

$0.70[0.68 ; 0.71] \quad 2.6 \%$

$0.65 \quad[0.62 \cdot 0.68] \quad 26 \%$

$0.79[0.77 ; 0.81] \quad 2.6 \%$

$0.76[0.74 ; 0.78] \quad 2.6 \%$

$0.83[0.82 ; 0.85] \quad 2.6 \%$

$0.68 \quad[0.65 ; 0.71] \quad 2.6 \%$

$0.55[0.52 ; 0.59] \quad 2.6 \%$

$0.72[0.70 ; 0.73] \quad 2.6 \%$

$0.74[0.72 ; 0.76] \quad 2.6 \%$

$0.54[0.51 ; 0.57] \quad 2.6 \%$

$0.76[0.75 ; 0.77] \quad 2.6 \%$

$0.45[0.44 ; 0.46] \quad 2.6 \%$

$0.40 \quad[0.37 ; 0.43] \quad 2.6 \%$

$0.35[0.32 ; 0.37] \quad 2.6 \%$

$0.69[0.67 ; 0.71] \quad 2.6 \%$

$0.91 \quad[0.90 ; 0.92] \quad 2.6 \%$

$0.86[0.82 ; 0.90] \quad 2.5 \%$

$0.72[0.71 ; 0.72] \quad 2.6 \%$

$0.69[0.66 ; 0.71] \quad 2.6 \%$

$0.92[0.90 ; 0.94] \quad 25 \%$

$0.85[0.83 ; 0.86] \quad 2.6 \%$

$0.78[0.74 ; 0.81] \quad 2.5 \%$

$0.86[0.86 ; 0.86] \quad 2.6 \%$

$0.63[0.60 ; 0.66] \quad 2.6 \%$

$0.70[0.68 ; 0.72] \quad 2.6 \%$

$0.95[0.94 ; 0.95] \quad 2.6 \%$

$0.77[0.76 ; 0.79] \quad 2.6 \%$

$0.74[0.70 ; 0.78] \quad 2.5 \%$

$0.93[0.92 ; 0.94] \quad 2.6 \%$

$0.70[0.68 ; 0.72] \quad 2.6 \%$

$0.97 \quad[0.97 ; 0.98] \quad 2.5 \%$

$0.84[0.83 ; 0.84] \quad 2.6 \%$

$0.85[0.85 ; 0.86] \quad 2.6 \%$

$0.76[0.76 ; 0.77] \quad 2.6 \%$

$0.75[0.71 ; 0.79] 100.0 \%$

Figure (6) Forest plot of the pooled vaccine acceptance 
medRxiv preprint doi: https://doi.org/10.1101/2021.05.15.21257261; this version posted May 18, 2021. The copyright holder for this preprint

\section{Discussion}

The vaccine for COVID-19 availability is a critical step to face the COVID-19 pandemic. But vaccine hesitancy represents a great threat to global health during this pandemic and limits the power of health systems to control the COVID-19 pandemic. Hence, estimating the COVID-19 vaccine hesitancy represents a tool to design an action plan to improve the vaccine acceptance.

In this meta-analysis, there was large variability between the studies discussing COVID-19 hesitancy in terms of vaccine acceptance. We aimed to determine the proportion of the population who are rejecting and accepting COVID-19 vaccine worldwide. We included 39 cross- sectional surveys conducted in 21 countries requiting 173213 participants. The quality of studies ranged from unsatisfactory, to very good. Our meta-analysis revealed that the pooled proportion of COVID-19 vaccine hesitancy was 17\% (95\% CI: 14-20) while the pooled proportion of COVID-19 vaccine acceptance was 75\% (95\% CI: 71-79). The main reported determinant of vaccine acceptance was case fatality and number of reported cases. Time effect was not associated with vaccine acceptance.

In the same line, a rapid systematic review and meta-analysis on COVID 19 vaccine hesitancy was conducted by Ronbinson et al, (61) to estimate the proportion of individuals refusing COVID-19 vaccine in 13 countries among 58,656 individuals. They reported that about $20 \%$ of the participants refused COVID-19 vaccine. They observed that differences across countries were very substantial and resulted in a heterogeneity above $90 \%$. Furthermore, they declared that the trend of rejection increased with time. The main determinants of COVID19 vaccine rejection were being female, of low educational level, or belonging to minor ethnicity.

Another review conducted by Lin et al, (62) compared trends in vaccination receptivity over time across US and international polls. The data sources included academic literature, news and official reports published by 20 October 2020. A total of 126 studies and surveys were included. The authors reported that there was a noticeable decline in vaccine acceptance (from $>70 \%$ in March to $<50 \%$ in October) with demographic, socioeconomic, and partisan divides observed. Perceived risk, concerns over vaccine safety and effectiveness, doctors' recommendations, and inoculation history 
medRxiv preprint doi: https://doi.org/10.1101/2021.05.15.21257261; this version posted May 18, 2021. The copyright holder for this preprint

were common factors. Impacts of regional infection rates, gender, and personal COVID-19 experience were inconclusive. Unique COVID-19 factors included political party orientation, doubts toward expedited development/approval process, and perceived political interference. Many receptive participants preferred to wait until others have taken the vaccine; mandates could increase resistance.

We speculate that the difference in vaccine acceptance may be affected by vaccine efficacy and side effects. Vaccines' side effects range between local to systemic, and short to long term events. The reported common side effects are generally mild to moderate and last for a few days. These include injection site pain, fatigue, rigors, fever, muscle and joints pains. Less commonly, a vaccine recipient may develop allergic reaction or anaphylaxis, and neurological side effects; however they are rarely reported (63). There is a rising concern particularly related to reported thrombo-embolic events, particularly after administration of AstraZeneca vaccine in Europe, but the European Medicines Agency concluded that the benefits of the vaccine overweighs the potential risk of this rare side effect (64). In this context, Kaplan et al, (65) underlined that vaccine acceptance improved when vaccine efficacy exceeds 70\%. Moreover, they addressed that minor side effects, such as a sore arm or fever lasting for a day did not affect vaccine acceptance, while major side effects in 1/100000 greatly affected vaccine acceptance. These side effects may vary according to the type of vaccine used in each country. Emerging evidence suggests that both exposure to misinformation about COVID-19 and public concerns over the safety of vaccines may be contributing to the observed decline in intentions to be vaccinated, and this highlights the need for measures to address public acceptability, trust and concern over the safety and benefit of approved vaccines $(66,67)$. This finding highlights the power of social media. Some studies emerged in the last months discussing the vaccine confidence in several populations, especially in countries with high burden of diseases like Pakistan (68). The role of recent misinformation was evident in the study of Loomba et al, (Measuring the impact of COVID-19 vaccine misinformation on vaccination intent in the UK and USA), it induced a decline in intent of 6.2 percentage points in the UK and 6.4 percentage points in the USA, among those who stated that they would definitely accept a vaccine. From another perspective, other studies analyzed attitudes toward COVID vaccination like the impact of 
medRxiv preprint doi: https://doi.org/10.1101/2021.05.15.21257261; this version posted May 18, 2021. The copyright holder for this preprint

education, whether medical or nonmedical students, on their decision (35).With the development of multiple effective vaccines, Immunization programs are only successful when there are high rates of acceptance and coverage (69). To accomplish this, it is critical to understand vaccine-acceptance messaging to effectively control the pandemic and prevent thousands of additional deaths (70).Individuals commonly considered COVID-19 to be a very severe disease, although they expected to experience less severe symptoms themselves. Individuals also worried more about transmitting the disease to others than about falling ill personally (71).

The strongest predictor of intentions to accept a COVID-19 vaccine recommended by authorities was the degree to which respondents trusted the vaccine to be safe. Perceived vaccine safety explained $52 \%$ of the variance in intentions to vaccinate (72). The study of Malik et al. shows that COVID-19 vaccine acceptance can be predicted with relatively high accuracy by readily available demographic characteristics. Since the beginning of the COVID-19 pandemic in the United States, it has been clear that low-income and communities of color are at higher risk for infection and death from COVID-19 (22).

\section{Strengths and limitations}

One of the main strength points in this study is the search strategy, we searched 12 different databases. Each citation was screened by two reviewers and disagreement was solved by a senior author. The same was done for quality assessment to ensure robust evidence. A large proportion of the included studies used quota (as opposed to probability-based sampling) and were pre-prints yet to be peer reviewed (as opposed to published journal articles). However, the type of sampling method used (quota vs. probability) had minimal impact on intentions estimates and that studies reported in pre-prints produced similar effect estimates as peer-reviewed journals. One of the main limitations was different tools used to assess vaccine acceptance in addition, the data collected either through face-to-face interview or through online data collection tools. We think that this may affect the internal validity of the study. However, we segregated analysis based on the method of data collection and the difference was not significant. 


\section{Conclusions}

COVID-19 vaccine rejection is low; however, continuous health education and social support is necessary to maintain the high acceptance rates. Time and residency have no significant effect on vaccine acceptance. However, the country-level case fatality and the officially reported number of cases were significant predictors of COVID-19 vaccine acceptance. That's why encouraging the health authorities to accurately follow \& announce case fatalities could be a major contributing factor to increasing vaccine acceptance. We believe that this study will demonstrate public hesitancy and help further development of motivational interview sessions and community-based education tailored according to the population education and individual concerns (73).Although, the main predictor for covid 19 vaccine acceptance or rejection is reporting transparency statement, there are poor transparency of documented information that guide the public decision regarding the vaccine acceptance.Global Health care authorities must report and announce for all transparency international freedom of information act templates ( FOIA) to the public for requiring vaccine and providing accurate information regarding all types of vaccines.(74) 
medRxiv preprint doi: https://doi.org/10.1101/2021.05.15.21257261; this version posted May 18, 2021. The copyright holder for this preprint (which was not certified by peer review) is the author/funder, who has granted medRxiv a license to display the preprint in perpetuity.

It is made available under a CC-BY 4.0 International license .

\section{REFERENCES}

1. Centers for Disease Control Prevention. Ten great public health achievements--United States, 1900-1999. MMWR Morbidity and mortality weekly report. 1999;48(12):241-3.

2. Fine $P$, Eames K, Heymann DL. "Herd immunity": a rough guide. Clinical infectious diseases. 2011;52(7):911-6.

3. Thangaraju P, Venkatesan S. WHO Ten threats to global health in 2019: Antimicrobial resistance. Cukurova Medical Journal. 2019;44(3):1150-1.

4. MacDonald NE. Vaccine hesitancy: Definition, scope and determinants. Vaccine. 2015;33(34):4161-4.

5. World Health Organization. Meeting of the Strategic Advisory Group of Experts on immunization, October 2014-conclusions and recommendations. Weekly Epidemiological Record= Relevé épidémiologique hebdomadaire. 2014;89(50):561-76.

6. Pang J, Wang MX, Ang IYH, Tan SHX, Lewis RF, Chen JI-P, et al. Potential rapid diagnostics, vaccine and therapeutics for 2019 novel coronavirus (2019-nCoV): a systematic review. Journal of clinical medicine. 2020;9(3):623.

7. World Health Organization. WHO Director-General's opening remarks at the media briefing on COVID-19 - 12 October 20202021 [3 May 2021]. Available from:

https://www.who.int/director-general/speeches/detail/who-director-general-s-opening-remarks -at-the-media-briefing-on-covid-19---12-october-2020.

8. World Health Organization. Coronavirus disease (COVID-19): Herd immunity, lockdowns and COVID-19 2021 [Available from:

https://www.who.int/news-room/q-a-detail/herd-immunity-lockdowns-and-covid-19?gclid=CjOK CQjwvr6EBhDOARIsAPpqUPExiCZc6iOzRwcSQbOO-f2ls0aBxcd9JuTewyqwzxmb3slgBE-1s1waAqsz EALw wcB\#. Assessed on 3 May 2021.

9. Dong M, He F, Deng Y. How to Understand Herd Immunity in the Context of COVID-19. Viral immunology. 2021;34(3):174-81.

10. Corum J, Grady D, Wee S-L, Zimmer C. Coronavirus vaccine tracker. The New York Times. 2020;5.

11. Lazarus JV, Ratzan SC, Palayew A, Gostin LO, Larson HJ, Rabin K, et al. A global survey of potential acceptance of a COVID-19 vaccine. Nature medicine. 2021;27(2):225-8.

12. Puri N, Coomes EA, Haghbayan H, Gunaratne K. Social media and vaccine hesitancy: new updates for the era of COVID-19 and globalized infectious diseases. Human Vaccines \& Immunotherapeutics. 2020:1-8.

13. Higgins JP, Thomas J, Chandler J, Cumpston M, Li T, Page MJ, et al. Cochrane handbook for systematic reviews of interventions: John Wiley \& Sons; 2019.

14. Tricco AC, Lillie E, Zarin W, O'Brien KK, Colquhoun H, Levac D, et al. PRISMA extension for scoping reviews (PRISMA-SCR): checklist and explanation. Annals of internal medicine. 2018;169(7):467-73.

15. Wells GA, Shea B, O'Connell Da, Peterson J, Welch V, Losos M, et al. The Newcastle-Ottawa Scale (NOS) for assessing the quality of nonrandomised studies in meta-analyses. Oxford; 2000.

16. Johns Hopkins University. COVID-19 Dashboard by the Center for Systems Science and Engineering (CSSE) at Johns Hopkins University (JHU) 2021 [7 May 2021]. Available from: https://coronavirus.jhu.edu/map.html.

17. World Health Organization. Coronavirus disease (COVID-19) Weekly Epidemiological Update and Weekly Operational Update 2021 [7 May 2021]. Available from: https://www.who.int/emergencies/diseases/novel-coronavirus-2019/situation-reports/. 
medRxiv preprint doi: https://doi.org/10.1101/2021.05.15.21257261; this version posted May 18, 2021. The copyright holder for this preprint (which was not certified by peer review) is the author/funder, who has granted medRxiv a license to display the preprint in perpetuity.

It is made available under a CC-BY 4.0 International license.

18. Sharun K, Rahman CF, Haritha C, Jose B, Tiwari R, Dhama K. Covid-19 vaccine acceptance: Beliefs and barriers associated with vaccination among the general population in india. Journal of Experimental Biology and Agricultural Sciences. 2020;8(Special Issue 1).

19. Paul E, Steptoe A, Fancourt D. Attitudes towards vaccines and intention to vaccinate against COVID-19: Implications for public health communications. The Lancet Regional Health Europe. 2021;1:100012.

20. Kwok KO, Li K-K, Wei WI, Tang A, Wong SYS, Lee SS. Influenza vaccine uptake, COVID-19 vaccination intention and vaccine hesitancy among nurses: A survey. International journal of nursing studies. 2021;114:103854.

21. Wang K, Wong ELY, Ho KF, Cheung AWL, Chan EYY, Yeoh EK, et al. Intention of nurses to accept coronavirus disease 2019 vaccination and change of intention to accept seasonal influenza vaccination during the coronavirus disease 2019 pandemic: A cross-sectional survey. Vaccine. 2020;38(45):7049-56.

22. Malik AA, McFadden SM, Elharake J, Omer SB. Determinants of COVID-19 vaccine acceptance in the US. EClinicalMedicine. 2020;26:100495.

23. Taylor S, Landry CA, Paluszek MM, Groenewoud R, Rachor GS, Asmundson GJ. A proactive approach for managing COVID-19: The importance of understanding the motivational roots of vaccination hesitancy for SARS-CoV2. Frontiers in Psychology. 2020;11:2890.

24. Al-Mohaithef M, Padhi BK. Determinants of COVID-19 Vaccine Acceptance in Saudi Arabia: A Web-Based National Survey. Journal of multidisciplinary healthcare. 2020;13:1657-63. 25. Unroe KT, Evans R, Weaver L, Rusyniak D, Blackburn J. Willingness of long-term care staff to receive a COVID-19 vaccine: A single state survey. Journal of the American Geriatrics Society. 2020.

26. Murphy J, Vallières F, Bentall RP, Shevlin M, McBride O, Hartman TK, et al. Psychological characteristics associated with COVID-19 vaccine hesitancy and resistance in Ireland and the United Kingdom. Nature communications. 2021;12(1):1-15.

27. Salali GD, Uysal MS. COVID-19 vaccine hesitancy is associated with beliefs on the origin of the novel coronavirus in the UK and Turkey. Psychological medicine. 2020:1-3.

28. Fisher KA, Bloomstone SJ, Walder J, Crawford S, Fouayzi H, Mazor KM. Attitudes toward a potential SARS-CoV-2 vaccine: a survey of US adults. Annals of internal medicine. 2020;173(12):964-73.

29. La Vecchia C, Negri E, Alicandro G, Scarpino V. Attitudes towards influenza vaccine and a potential COVID-19 vaccine in Italy and differences across occupational groups, September 2020. 2020.

30. Sherman SM, Smith LE, Sim J, Amlôt R, Cutts M, Dasch H, et al. COVID-19 vaccination intention in the UK: results from the COVID-19 vaccination acceptability study (CoVAccS), a nationally representative cross-sectional survey. Human vaccines \& immunotherapeutics. 2020:1-10.

31. Lucia VC, Kelekar A, Afonso NM. COVID-19 vaccine hesitancy among medical students. Journal of Public Health. 2020.

32. Kreps S, Prasad S, Brownstein JS, Hswen Y, Garibaldi BT, Zhang B, et al. Factors associated with US adults' likelihood of accepting COVID-19 vaccination. JAMA network open. 2020;3(10):e2025594-e.

33. Gagneux-Brunon A, Detoc M, Bruel S, Tardy B, Rozaire O, Frappe P, et al. Intention to get vaccinations against COVID-19 in French healthcare workers during the first pandemic wave: a cross-sectional survey. Journal of Hospital Infection. 2021;108:168-73. 
medRxiv preprint doi: https://doi.org/10.1101/2021.05.15.21257261; this version posted May 18, 2021. The copyright holder for this preprint (which was not certified by peer review) is the author/funder, who has granted medRxiv a license to display the preprint in perpetuity.

It is made available under a CC-BY 4.0 International license .

34. Lin Y, Hu Z, Zhao Q, Alias H, Danaee M, Wong LP. Understanding COVID-19 vaccine demand and hesitancy: A nationwide online survey in China. PLoS neglected tropical diseases. 2020;14(12):e0008961.

35. Barello S, Nania T, Dellafiore F, Graffigna G, Caruso R. 'Vaccine hesitancy'among university students in Italy during the COVID-19 pandemic. European journal of epidemiology. 2020;35(8):781-3.

36. Dror AA, Eisenbach N, Taiber S, Morozov NG, Mizrachi M, Zigron A, et al. Vaccine hesitancy: the next challenge in the fight against COVID-19. European journal of epidemiology. 2020;35(8):775-9.

37. Akarsu B, Canbay Özdemir D, Ayhan Baser D, Aksoy H, Fidancı İ, Cankurtaran M. While studies on COVID-19 vaccine is ongoing, the public's thoughts and attitudes to the future COVID-19 vaccine. International journal of clinical practice. 2021;75(4):e13891.

38. Freeman D, Loe BS, Chadwick A, Vaccari C, Waite F, Rosebrock L, et al. COVID-19 vaccine hesitancy in the UK: the Oxford coronavirus explanations, attitudes, and narratives survey (Oceans) II. Psychological medicine. 2020:1-15.

39. Butter S, McGlinchey E, Berry E, Armour C. Psychological, social, and situational factors associated with COVID-19 vaccination intentions: A study of UK key workers and non-key workers. 2020.

40. Niankara I, Muqattash R, Traoret RI. Survey data for COVID-19 Vaccine preference analysis in the United Arab Emirates. Mendeley Data, Online v1 URL https://data mendeley com/datasets/pysxmjpkr4/1. 2020.

41. Ward JK, Alleaume C, Peretti-Watel P, Peretti-Watel P, Seror V, Cortaredona S, et al. The French public's attitudes to a future COVID-19 vaccine: The politicization of a public health issue. Social Science \& Medicine. 2020;265:113414.

42. Goldman RD, Yan TD, Seiler M, Cotanda CP, Brown JC, Klein EJ, et al. Caregiver willingness to vaccinate their children against COVID-19: Cross sectional survey. Vaccine. 2020;38(48):7668-73.

43. Reiter PL, Pennell ML, Katz ML. Acceptability of a COVID-19 vaccine among adults in the United States: How many people would get vaccinated? Vaccine. 2020;38(42):6500-7.

44. Wang J, Jing R, Lai X, Zhang H, Lyu Y, Knoll MD, et al. Acceptance of COVID-19 Vaccination during the COVID-19 Pandemic in China. Vaccines. 2020;8(3):482.

45. Kose S, Mandiracioglu A, Sahin S, Kaynar T, Karbus O, Ozbel Y. Vaccine hesitancy of the COVID-19 by health care personnel. International Journal of Clinical Practice. 2021;75(5):e13917.

46. Biasio LR, Bonaccorsi G, Lorini C, Pecorelli S. Assessing COVID-19 vaccine literacy: a preliminary online survey. Human vaccines \& immunotherapeutics. 2021;17(5):1304-12.

47. Grüner S, Krüger F. The intention to be vaccinated against COVID-19: stated preferences before vaccines were available. Applied Economics Letters. 2020:1-5.

48. Edwards B, Biddle N, Gray M, Sollis K. COVID-19 vaccine hesitancy and resistance: Correlates in a nationally representative longitudinal survey of the Australian population. PloS one. 2021;16(3):e0248892.

49. Detoc M, Bruel S, Frappe P, Tardy B, Botelho-Nevers E, Gagneux-Brunon A. Intention to participate in a COVID-19 vaccine clinical trial and to get vaccinated against COVID-19 in France during the pandemic. Vaccine. 2020;38(45):7002-6.

50. Adebisi YA, Alaran AJ, Bolarinwa OA, Akande-Sholabi W, Lucero-Prisno DE. When it is available, will we take it? Public perception of hypothetical COVID-19 vaccine in Nigeria. medRxiv. 2020. 
medRxiv preprint doi: https://doi.org/10.1101/2021.05.15.21257261; this version posted May 18, 2021. The copyright holder for this preprint (which was not certified by peer review) is the author/funder, who has granted medRxiv a license to display the preprint in perpetuity.

It is made available under a CC-BY 4.0 International license.

51. Barry M, Temsah M-H, Alhuzaimi A, Alamro N, Al-Eyadhy A, Aljamaan F, et al. COVID-19 vaccine confidence and hesitancy among healthcare workers: a cross-sectional survey from a MERS-CoV experienced nation. medRxiv. 2020.

52. Chen M, Li Y, Chen J, Wen Z, Feng F, Zou H, et al. An online survey of the attitude and willingness of Chinese adults to receive COVID-19 vaccination. Human Vaccines \& Immunotherapeutics. 2021:1-10.

53. Meyer MN, Gjorgjieva T, Rosica D. Healthcare worker intentions to receive a COVID-19 vaccine and reasons for hesitancy: A survey of 16,158 health system employees on the eve of vaccine distribution. medRxiv. 2020.

54. Robertson E, Reeve KS, Niedzwiedz CL, Moore J, Blake M, Green M, et al. Predictors of COVID-19 vaccine hesitancy in the UK household longitudinal study. Brain, behavior, and immunity. 2021.

55. Kerr JR, Schneider CR, Recchia G, Dryhurst S, Sahlin U, Dufouil C, et al. Predictors of COVID-19 vaccine acceptance across time and countries. medRxiv. 2020.

61. Robinson E, Jones A, Daly M. International estimates of intended uptake and refusal of COVID-19 vaccines: A rapid systematic review and meta-analysis of large nationally representative samples. medRxiv. 2020.

62. Lin C, Tu P, Beitsch LM. Confidence and Receptivity for COVID-19 Vaccines: A Rapid Systematic Review. Vaccines. 2021;9(1):16.

63. WHO.int. Side Effects of COVID-19 Vaccines 2021 [Accessed on 4th May 2021]. Available from: https://www.who.int/news-room/feature-stories/detail/side-effects-of-covid-19-vaccines

64. European Medicines Agency. AstraZeneca's COVID-19 vaccine: EMA finds possible link to very rare cases of unusual blood clots with low blood platelets 2021 [Available from:

https://www.ema.europa.eu/en/news/astrazenecas-covid-19-vaccine-ema-finds-possible-link-ve ry-rare-cases-unusual-blood-clots-low-blood Accessed on: 4th May 2021].

65. Kaplan RM, Milstein A. Influence of a COVID-19 vaccine's effectiveness and safety profile on vaccination acceptance. Proceedings of the National Academy of Sciences. 2021;118(10).

66. Daly M. Willingness to vaccinate against COVID-19 in the US: 3 Longitudinal evidence from a nationally representative sample of adults from April-4 October 20205.

67. Roozenbeek J, Schneider CR, Dryhurst S, Kerr J, Freeman AL, Recchia G, et al. Susceptibility to misinformation about COVID-19 around the world. Royal Society open science. 2020;7(10):201199.

68. Khan YH, Mallhi TH, Alotaibi NH, Alzarea Al, Alanazi AS, Tanveer N, et al. Threat of COVID-19 vaccine hesitancy in Pakistan: the need for measures to neutralize misleading narratives. The American journal of tropical medicine and hygiene. 2020;103(2):603-4.

69. Saied SM, Saied EM, Kabbash IA, Abdo SAEF. Vaccine hesitancy: Beliefs and barriers associated with COVID-19 vaccination among Egyptian medical students. Journal of medical virology. 2021.

70. Tran VD, Pak TV, Gribkova El, Galkina GA, Loskutova EE, Dorofeeva VV, et al.

Determinants of COVID-19 vaccine acceptance in a high infection-rate country: a cross-sectional study in Russia. Pharmacy Practice (Granada). 2021;19(1).

71. Park T, Ju I, Ohs JE, Hinsley A. Optimistic bias and preventive behavioral engagement in the context of COVID-19. Research in Social and Administrative Pharmacy. 2021;17(1):1859-66.

72. Latkin C, Dayton LA, Yi G, Konstantopoulos A, Park J, Maulsby C, et al. COVID-19 vaccine intentions in the United States, a social-ecological framework. Vaccine. 2021;39(16):2288-94.

73. European Centre for Disease Prevention \& Control. Catalogue of interventions addressing vaccine hesitancy: ECDC Stockholm; 2017 [ 
medRxiv preprint doi: https://doi.org/10.1101/2021.05.15.21257261; this version posted May 18, 2021. The copyright holder for this preprint (which was not certified by peer review) is the author/funder, who has granted medRxiv a license to display the preprint in perpetuity.

It is made available under a CC-BY 4.0 International license .

74. Transparency.2021.Transparency International the global coalition against corruption.[online]. Available at:<https://www.transparency.org/en/news/covid-19-vaccine-transparency>[Accessed 13 May 2021]. 